\title{
Avaliação da exposição dos médicos à radiação em procedimentos hemodinâmicos intervencionistas*
}

\author{
Evaluation of radiation exposure to physicians during hemodynamic interventional procedures
}

\author{
Leonardo Peres da Silva ${ }^{1}$, Claudia Lúcia de Pinho Maurício² ${ }^{2}$ Lucía Viviana Canevaro ${ }^{3}$, \\ Paulo Sérgio Oliveira ${ }^{4}$
}

Resumo OBJETIVO: Este trabalho avalia a distribuição de dose recebida por médicos envolvidos em procedimentos hemodinâmicos de angioplastia coronária e coronariografia. A influência de alguns fatores, como o modo de fluoroscopia pulsado ou contínuo e o local de acesso à veia e/ou artéria, foi investigada. MATERIAIS E MÉTODOS: Para esta avaliação foram feitas medições utilizando dosímetros termoluminescentes de LiF:Mg, Ti, posicionados em sete diferentes pontos do corpo dos profissionais: mãos, joelho, pescoço, testa e tórax, por dentro e por fora do avental de chumbo. A dose foi avaliada, por exame, nos médicos que executaram os procedimentos (30 de angioplastia e 60 de coronariografia). Os dosímetros termoluminescentes foram calibrados na grandeza operacional equivalente de dose pessoal, $H_{p}(d)$, nas profundidades de 0,07, 3 e $10 \mathrm{~mm}$. RESULTADOS: Os resultados mostram a importância do uso do protetor de tireóide e avental de chumbo para a redução da dose recebida pelos médicos. As doses dos profissionais que executaram procedimentos por via braquial usando modo contínuo de fluoroscopia foram mais altas do que os que executaram por via femoral e modo pulsado de fluoroscopia. CONCLUSÃO: Este estudo mostra a necessidade de medidas adicionais de proteção e a implementação de mecanismos de treinamento em proteção radiológica para os médicos que trabalham com cardiologia intervencionista.

Unitermos: Radiologia intervencionista; Fluoroscopia; Procedimentos hemodinâmicos; Proteção radiológica; Dosimetria termoluminescente.

Abstract OBJECTIVE: The present study evaluates the dose distribution received by physicians involved in hemodynamic procedures of coronary angiography and coronariography. The influence of some factors such as pulsed or continuous fluoroscopy mode and vein and/or artery access site was investigated. MATERIALS AND METHODS: Measurements have been performed with LiF:Mg,Ti thermoluminescent dosimeters placed on seven different sites of the practitioners' bodies: hands, left knee, neck, forehead and chest, under and over the lead apron. Radiation doses for each procedure were evaluated in physicians who had performed 60 coronariography and $\mathbf{3 0}$ angioplasty procedures. The thermoluminescent dosimeters were calibrated in the operational quantity personal dose equivalent, $H_{p}(d)$, at depths of $0.07,3$ and $10 \mathrm{~mm}$. RESULTS: The results of the present study demonstrate the significant role played by thyroid protectors and lead aprons for reducing radiation doses received by practitioners. Occupational doses in continuous mode fluoroscopy through brachial access were higher than with pulsed mode fluoroscopy through femoral access. CONCLUSION: The present study has demonstrated the necessity of implementing additional protective measures as well as a mechanism of training in radiation protection for physicians involved in interventional cardiological procedures.

Keywords: Interventional radiology; Fluoroscopy; Hemodynamic procedures; Radiation protection; Thermoluminescent dosimetry.

Silva LP, Maurício CLP, Canevaro LV, Oliveira PS. Avaliação da exposição dos médicos à radiação em procedimentos hemodinâmicos intervencionistas. Radiol Bras. 2008;41(5):319-323.

\footnotetext{
* Trabalho realizado no Instituto Nacional de Cardiologia Laranjeiras (INCL), Rio de Janeiro, RJ, Brasil.

1. Mestre, Físico Médico em Radioterapia, Professor Substituto do Departamento de Radiologia da Faculdade de Medicina da Universidade Federal do Rio de Janeiro (UFRJ), Rio de Janeiro, RJ, Brasil.

2. Doutora, Chefe do Serviço de Monitoração Individual Ex terna do Instituto de Radioproteção e Dosimetria - Comissão Nacional de Energia Nuclear (IRD-CNEN), Rio de Janeiro, RJ, Brasil.

3. Doutora, Pesquisadora do Departamento de Física Médica do Instituto de Radioproteção e Dosimetria - Comissão Nacional de Energia Nuclear (IRD-CNEN), Rio de Janeiro, RJ, Brasil.

4. Doutor, Chefe do Serviço de Hemodinâmica do Instituto Nacional de Cardiologia Laranjeiras (INCL), Rio de Janeiro, RJ, Brasil.
}

\section{INTRODUÇÃO}

A radiologia intervencionista comporta intervenções diagnósticas e/ou terapêuticas guiadas por acesso percutâneo. Geralmente, estes procedimentos são realizados sob

Endereço para correspondência: Dr. Leonardo Peres da Silva. Rua Doutor Bernardino, 111, ap. 202, Praça Seca. Rio de Janeiro, RJ, Brasil, 21320-020. E-mail: leo_ird@yahoo.com.br Recebido para publicação em 9/12/2007. Aceito, após revisão, em 5/3/2008. anestesia local e/ou sedação. A fluoroscopia é utilizada para localizar a lesão ou o local de tratamento, para monitorar o procedimento e para documentar a terapia ${ }^{(1)}$. Substâncias radiopacas, conhecidas como meios de contraste, são utilizadas para a visualização de órgãos ou tecidos radiotransparentes na tela do monitor.

Estes procedimentos são plenamente justificáveis, tanto para os pacientes quanto para o sistema de saúde, pois substituem 
cirurgias complicadas por procedimentos médicos mais simples, diminuindo o risco e o tempo de internação para o paciente, assim como o custo total do procedimento. Entretanto, a radiologia intervencionista é uma das especialidades da radiologia médica que proporciona as maiores doses aos profissionais $^{(2)}$. As doses ocupacionais são altas, já tendo sido identificados em médicos intervencionistas efeitos determinísticos da radiação, como catarata e epilação das extremidades ${ }^{(\mathbf{1}, \mathbf{3})}$. Essas doses elevadas são causadas pela proximidade de alguns membros da equipe, principalmente o médico intervencionista, ao paciente e ao tubo de raios X. Para diminuir a exposição devem ser utilizados anteparos móveis entre o médico e o paciente e vestimentas de proteção individual ${ }^{(\mathbf{4})}$. As principais vestimentas de proteção individual são: avental de chumbo, protetor de tireóide, óculos plumbíferos e luvas de materiais atenuadores.

É importante chamar a atenção para o fato de que profissionais que trabalham em salas de radiologia intervencionista são cardiologistas, ortopedistas, cirurgiões vasculares, neurologistas, etc., que, de maneira geral, não têm formação em proteção radiológica. Esta formação está se tornando condição imprescindível para o uso de equipamentos de raios $\mathrm{X}$ especialmente dedicados devido, entre outras razões, à progressiva sofisticação tecnológica.

No Brasil, a Portaria 453/98 do Ministério da Saúde ${ }^{(5)}$ estabelece as diretrizes de proteção radiológica para a radiologia médica e odontológica. Este regulamento estabelece os requisitos de proteção radiológica necessários para o licenciamento e controle dos serviços de radiologia, incluindo os que usam fluoroscopia. Seus princípios básicos são os usados internacionalmente e pela Comissão Nacional de Energia Nuclear $(\mathrm{CNEN})^{(6,7)}$ :

1. Justificação da prática e das exposições médicas individuais;

2. Otimização da proteção radiológica;

3. Limitação das doses individuais ocupacionais e de público.

A prática da radiologia intervencionista como meio diagnóstico e terapêutico é plenamente justificada; no entanto, aspectos relativos à sua otimização ainda devem ser estudados, avaliados e implementados. A Portaria 453/98 regulamenta, principal- mente, os aspectos relacionados à prática da radiologia convencional e não apresenta exigências específicas para a radiologia intervencionista.

Para acompanhar as condições de trabalho dos profissionais é exigida a monitoração individual mensal de todos os trabalhadores de radiologia médica, com a finalidade de estimar sua dose efetiva e, em alguns casos, sua dose equivalente no cristalino e extremidades. A “dose efetiva” é a grandeza que estima o risco total da indução de efeitos estocásticos provenientes de uma exposição à radiação. A "dose equivalente em um tecido ou órgão" é a dose absorvida média nesse tecido ou órgão, multiplicada por um fator de ponderação relacionado ao tipo de radiação. Para fótons, esse fator de ponderação é igual a 1. A dose efetiva média anual recebida pelo trabalhador não deve exceder $20 \mathrm{mSv}$ em qualquer período de cinco anos consecutivos, não podendo ultrapassar $50 \mathrm{mSv}$ em nenhum ano. A dose equivalente anual não deve exceder $500 \mathrm{mSv}$ para a pele e extremidades e $150 \mathrm{mSv}$ para o cristalino. Doses efetivas acima de 1,5 mSv/mês devem ser investigadas.

Quando é usado avental de chumbo, o dosímetro individual de tórax deve ser colocado sobre o avental, na parte mais exposta do tórax, dividindo-se o valor de sua medição por um fator de correção igual a 10, para estimar a dose efetiva. $\mathrm{O}$ valor de sua medição deve ser interpretado como a dose equivalente nas regiões não-blindadas do corpo. No caso de as extremidades e/ou olhos estarem sujeitos a doses significativamente maiores do que a leitura do dosímetro do tórax, dosímetros adicionais devem ser usados. Todas as medições devem ser realizadas usando-se a grandeza "equivalente de dose pessoal", $\mathrm{H}_{\mathrm{p}}(\mathrm{d})$, que é a grandeza operacional de monitoração individual externa, definida como a dose absorvida em um ponto a uma profundidade $d$, medida em milímetros, do corpo, no tecido mole, multiplicada pelo fator de qualidade da radiação, que no caso dos raios $\mathrm{X}$ é igual a 1 . No tórax, utiliza-se $d=10 \mathrm{~mm}$; nas extremidades, $d=0,07 \mathrm{~mm}$; e para o cristalino, $d=3 \mathrm{~mm}$.

Atualmente, a radiologia intervencionista é amplamente utilizada em cardiologia, sendo denominada cardiologia inter- vencionista. As doses recebidas pelos profissionais que atuam em cardiologia intervencionista dependem de vários fatores, como características clínicas e técnicas de cada procedimento, experiência do médico intervencionista, treinamento em proteção radiológica, uso de vestimentas de proteção individual, conhecimento do correto funcionamento do aparelho de raios $\mathrm{X}$, e a posição do médico em relação ao tubo de raios $\mathrm{X}$ e ao paciente.

No presente trabalho foi estudada a influência de alguns fatores na dose recebida pelos médicos intervencionistas em procedimentos de coronariografia e angioplastia coronária, com o objetivo de contribuir para a otimização da proteção radiológica ocupacional nesta atividade. Estes dois procedimentos foram escolhidos por serem os mais freqüentes no hospital analisado.

\section{MATERIAIS E MÉTODOS}

Foram acompanhados profissionais de três salas de hemodinâmica de um hospital-escola da cidade do Rio de Janeiro, durante a realização de exames de angioplastia coronária (30 procedimentos) e coronariografia (60 procedimentos). Durante esses exames, normalmente, permanecem dentro da sala: um médico responsável (sênior), um médico assistente (júnior), um enfermeiro e um técnico. Durante as angioplastias, além desses profissionais, sempre está presente um médico anestesista. Cada profissional realiza cerca de 1.000 procedimentos por ano.

Os procedimentos foram realizados nos seguintes equipamentos de raios $\mathrm{X}$ : dois Siemens Coroscop Hip Top (Siemens AG; Munique, Alemanha), trifásico, modos contínuo e pulsado de radiação com 7,5, 15 e $30 \mathrm{f} / \mathrm{s}$, tubo de raios $\mathrm{X}$ de até $150 \mathrm{kV}$, filtração adicional de $0,2 \mathrm{~mm}$ de cobre para fluoro, intensificador de imagem com campos de 13,17 e $23 \mathrm{~cm}^{2}$ e taxas de kerma no ar normal e alta (high), denominadas, respectivamente, fluoro e fluoro+; um GE Arcomax CGR (General Electric Medical Systems; Milwaukee, EUA), mesa Angix 80, trifásico, modo contínuo de radiação, com tubo de raios $\mathrm{X}$ de até $150 \mathrm{kV}$, taxas de kerma no ar baixa (low), normal e alta (high) e intensificador de imagem com campos de 15,23 e $30 \mathrm{~cm}^{2}$. 
Foram utilizados dosímetros termoluminescente (TLDs) do tipo TLD100 (LIF: Mg,Ti da Harshaw) em forma de chip de 3 $\times 3 \times 0,5 \mathrm{~mm}^{3}$, fornecidos pelo Laboratório de Dosimetria Termoluminescente (LDT) do Instituto de Radioproteção e Dosimetria (IRD). Esses dosímetros foram escolhidos, principalmente, por apresentarem baixa dependência energética, linearidade em toda a faixa de valores de doses esperados e boa estabilidade ${ }^{(\mathbf{8})}$.

Os dosímetros foram colocados em pequenos sacos plásticos pretos (para blindar a luz e proteger o TLD). Estes saquinhos plásticos foram fixados com esparadrapo em sete pontos do corpo dos profissionais que realizaram os procedimentos intervencionistas analisados: dois no tórax (por cima e por baixo do avental de chumbo), dois nas mãos (um no pulso da mão direita e um no pulso da mão esquerda), um na testa, um no joelho esquerdo e um no pescoço. A Figura 1 apresenta os sete pontos avaliados.

O dosímetro colocado no tórax por dentro do avental estima a dose nos órgãos blindados pelo avental. O ponto localizado na testa é usado para a estimativa de dose no cristalino, $\mathrm{H}_{\mathrm{p}}(3)$, o dosímetro do joelho estima a dose nas extremidades inferiores, $\mathrm{H}_{\mathrm{p}}(0,07)$, o das mãos estima a dose nas extremidades superiores, $\mathrm{H}_{\mathrm{p}}(0,07)$, e o do pescoço, a dose na tireóide, $\mathrm{H}_{\mathrm{p}}(10)$. Foram realizadas medições de dose na tireóide (pescoço) em profissionais que utilizaram protetores de tireóide e que não usaram esta proteção durante a realização dos procedimentos. No caso de o trabalhador usar protetor de tireóide, o TLD foi colocado por baixo deste. O valor medido pelo dosímetro colocado no tórax por fora do avental estima a dose nas partes não-blindadas do corpo e, dividindo sua resposta por 10 , a dose efetiva.

As medições foram realizadas por procedimento e somente um médico foi monitorado por procedimento (apenas aquele que executou o exame). No total foram avaliados cinco diferentes médicos em 30 procedimentos de angioplastia e 60 procedimentos de coronariografia.

Alguns procedimentos de coronariografia foram realizados no modo pulsado e outros foram efetuados no modo contínuo de fluoroscopia (imagem em tempo real). Em ambos os equipamentos de raios $\mathrm{X}$ o modo de aquisição de imagem (imagem gravada) foi digital; o aparelho GE Arcomax CGR foi adaptado para substituir o modo de aquisição de imagens de cine para o digital. Dessa maneira, foi possível realizar uma comparação das doses recebidas pelos profissionais nas mãos, olhos e joelhos nesses três modos.

Nos procedimentos descritos neste trabalho, alguns profissionais utilizaram o modo braquial (pelo braço) para o acesso à veia e/ou à artéria para a introdução do cateter, e outros o fizeram por via femoral (pela virilha). Foi feita comparação das doses recebidas pelos profissionais que realizaram esses procedimentos por esses dois modos de acesso.

\section{RESULTADOS}

Na Figura 2 apresenta-se a distribuição do valor medido para a grandeza $\mathrm{H}_{\mathrm{p}}(10)$ no pescoço dos profissionais que usaram ou não a proteção.

Na Figura 3 apresenta-se uma comparação dos resultados das medições realizadas com dosímetros colocados por dentro e por fora do avental.

Nas Figuras 4, 5, 6 e 7 apresenta-se a distribuição dos valores medidos dos equivalentes de dose nas mãos e nos olhos dos profissionais que executaram os procedimentos por via braquial e por via femoral e nos modos pulsado e contínuo de fluoroscopia.

Alguns procedimentos de coronariografia foram realizados com acesso por via braquial utilizando o modo contínuo. A Figura 8 apresenta os valores de equivalente de dose nas mãos, cristalino e joelho dos médicos que realizaram os procedimentos que combinaram as duas técnicas (modo contínuo + via braquial).

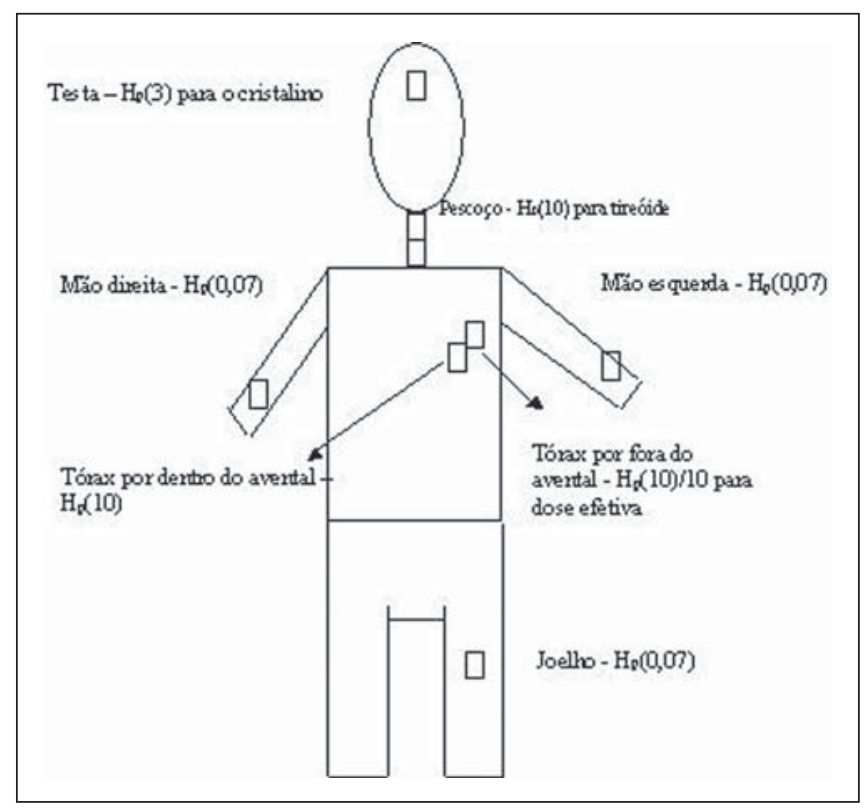

Figura 1. Pontos de monitoração dos profissionais.

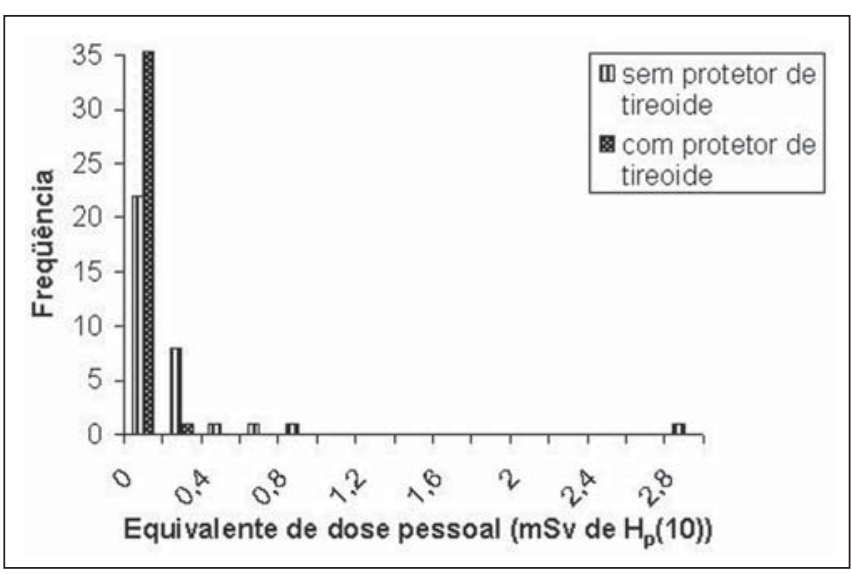

Figura 2. Análise dos valores de equivalente de dose pessoal na tireóide com o profissional usando o protetor ou não. 


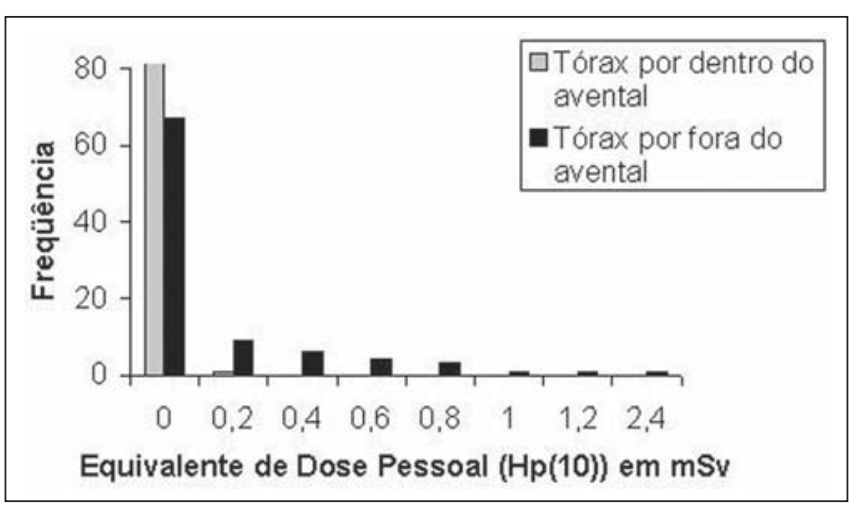

Figura 3. Análise dos valores de equivalente de dose pessoal por dentro e por fora do avental de chumbo.

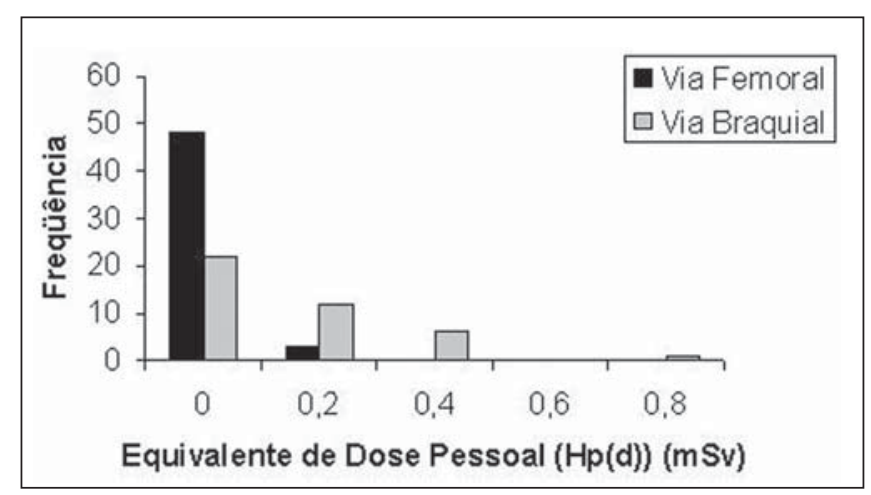

Figura 5. Análise do equivalente de dose no cristalino para os modos de acesso a artéria e/ou veia por via femoral e braquial.

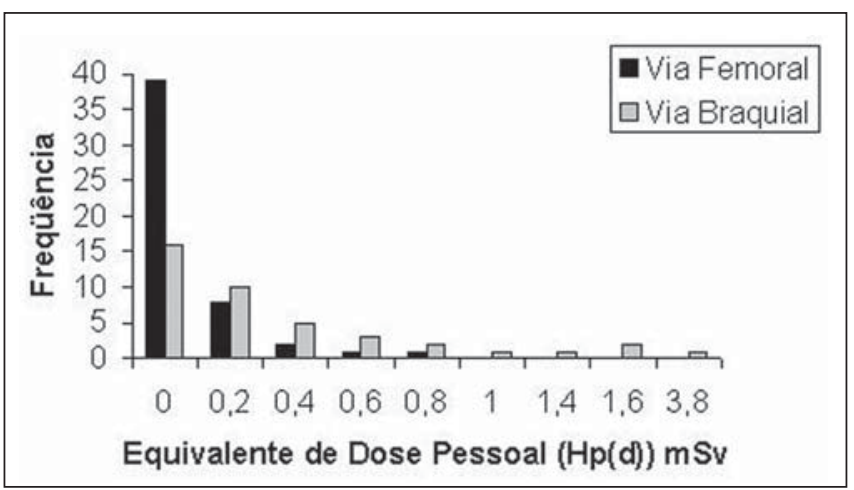

Figura 7. Análise do equivalente de dose nas mãos para os modos de acesso a artéria e/ou veia por via femoral e braquial.

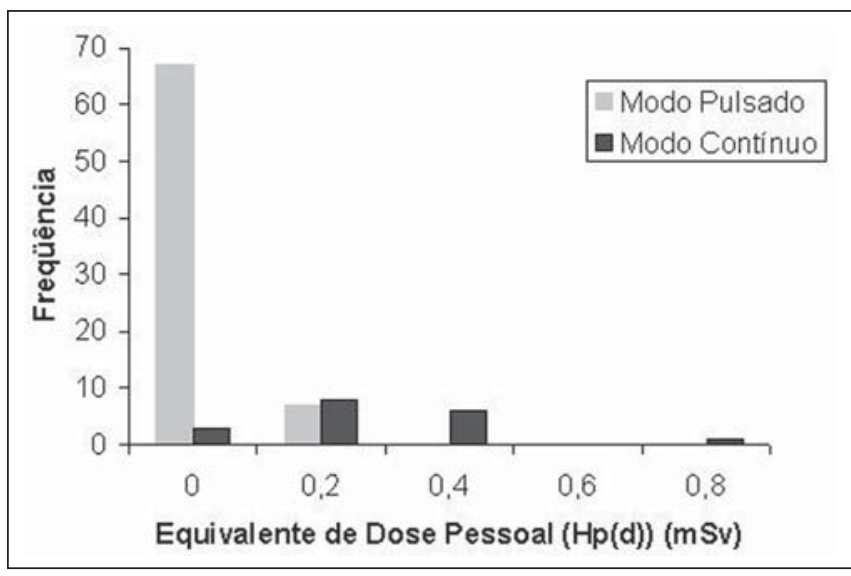

Figura 4. Análise do equivalente de dose no cristalino para os modos pulsado e contínuo de fluoroscopia.

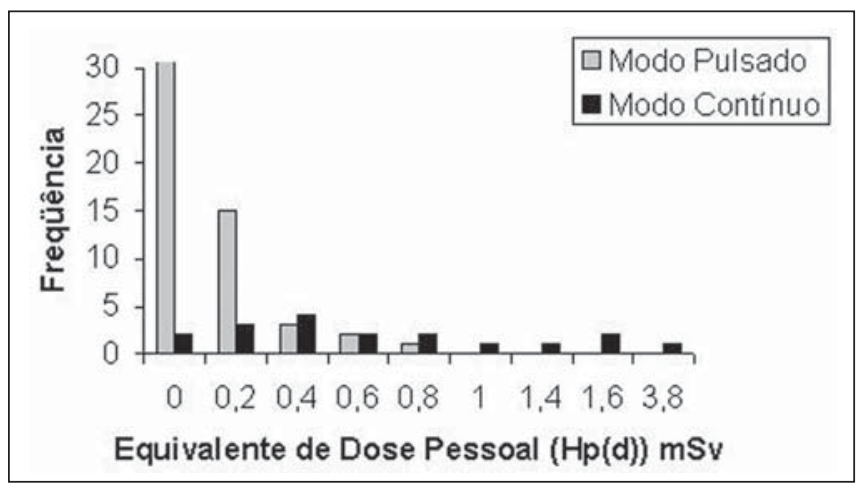

Figura 6. Análise do equivalente de dose nas mãos para os modos pulsado e contínuo de fluoroscopia.

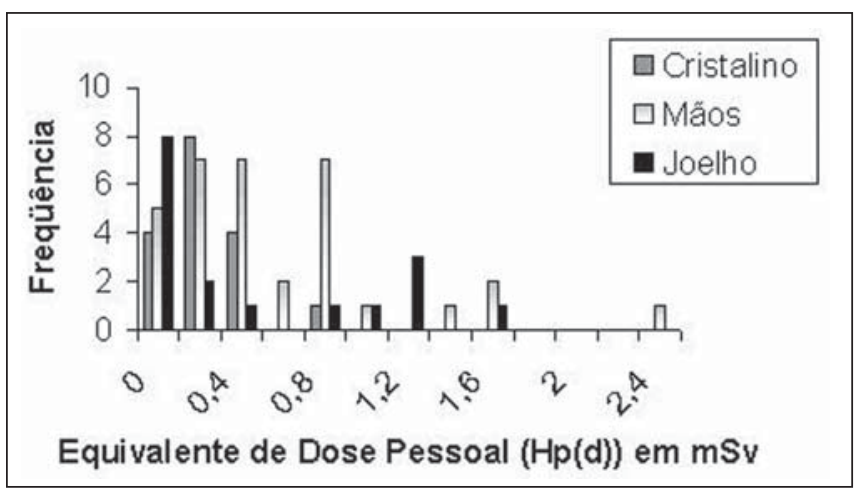

Figura 8. Equivalente de dose recebido pelos profissionais que realizaram os procedimentos no modo contínuo de fluoroscopia e por via braquial.

\section{DISCUSSÃO}

No caso da monitoração da tireóide dos médicos, os maiores valores de equivalente de dose registrados foram obtidos quando o profissional não usava o protetor de tireóide, mostrando claramente a importância do uso desses protetores para a redução da dose. O maior valor do equivalente de dose recebido pelos profissionais que usaram o protetor de tireóide foi de $0,3 \mathrm{mSv}$, enquanto sem o protetor esse valor alcançou, em um procedimento, $2,9 \mathrm{mSv}$.

A leitura do dosímetro por baixo do avental, na grandeza $\mathrm{H}_{\mathrm{p}}(10)$, representa a dose que o trabalhador recebeu neste ponto.
O objetivo desta comparação é verificar se a dose por fora do avental multiplicada pelo fator de atenuação do avental de 0,1 é próxima da dose por dentro ao avental. O maior valor de $\mathrm{H}_{\mathrm{p}}(10)$ medido por fora do avental foi de $2,4 \mathrm{mSv}$, que se for dividido por 10 (ou multiplicado por 0,1 ) resultará em um valor máximo de $0,24 \mathrm{mSv}$, que está 
na faixa de valores medidos pelo dosímetro por dentro do avental.

Os valores de dose nos olhos, mãos e joelho dos profissionais que realizaram os procedimentos descritos neste trabalho variaram muito conforme o local de acesso à veia e/ou à artéria, por onde são introduzidos os cateteres, e pelo modo de fluoroscopia utilizado (contínuo ou pulsado).

Os profissionais que realizaram os procedimentos por via braquial receberam doses maiores do que os que os executaram por via femoral. Este fato é decorrente do posicionamento do médico durante o procedimento realizado pelos dois modos. Por via braquial, o médico fica mais próximo do tubo de raios $\mathrm{X}$ do que por via femoral ${ }^{(\mathbf{9})}$.

Os valores de equivalente de dose avaliados nas mãos, olhos e joelhos dos profissionais que executaram os procedimentos de coronariografia no modo contínuo foram, em média, maiores do que os do modo pulsado de fluoroscopia.

Os valores de dose apresentados nas Figuras 4 a 8 chamam a atenção para a necessidade do uso de óculos plumbíferos para proteção do cristalino e de proteção adicional nas extremidades, principalmente as mãos, pois foram estas que receberam as maiores doses. Os valores das doses no cristalino e nas extremidades, quando multiplicados pela carga de trabalho anual, podem ultrapassar os limites de dose anual estabelecidos por norma para essas regiões anatômicas, podendo causar complicações futuras. Por exemplo, considerando a média das doses no cristalino sob essas condições, 0,35 mSv, e multiplicando por uma carga de trabalho de 1.500 procedimentos por ano, resulta em uma dose equivalente de 0,52 Sv, que ultrapassa o limiar de dose de $0,5 \mathrm{~Sv}^{(\mathbf{1 0})}$ para a formação de opacidades no cristalino.

O uso de apenas um único dosímetro no tórax é adequado, quando se considera que esta é a parte mais exposta do corpo. Uma vez que na cardiologia intervencionista outras partes do corpo estão sujeitas a doses maiores, como mostrado nas Figuras 4 a 8, principalmente as mãos, este trabalho recomenda o uso de dosímetros adicionais para essas regiões.

\section{CONCLUSÕES}

Os resultados mostram claramente a importância do uso do protetor de tireóide, além do avental de chumbo, por todos os médicos intervencionistas em todos os procedimentos.

A dose por fora do avental multiplicada pelo fator de atenuação do avental de 0,1 é próxima da dose por dentro do avental.

As doses nas mãos, cristalino e joelhos dos profissionais que executam procedimentos intervencionistas por via braquial e no modo contínuo de fluoroscopia foram mais altas do que os realizados por via femoral e no modo pulsado de fluoroscopia. As doses nesses pontos, quando multiplicadas pela carga anual de trabalho, podem ultrapassar os limites dessas regiões anatômicas estabelecidos por normas. Neste caso, é necessário adotar medidas adicionais de proteção. Para diminuir as doses dos médicos intervencionistas, se faz necessário implementar mecanismos de treinamento em proteção radiológica para os profissionais que trabalham com cardiologia intervencionista e realizar, periodicamente, o controle de qualidade nos equipamentos que emitem radiação ${ }^{(11)}$. É necessário conscientizar todos os envolvidos sobre a importância da utilização de outras vestimentas de proteção individual, além do avental de chumbo, tais como protetores de tireóide, óculos plumbíferos e luvas $^{(4)}$. Existem, no mercado, luvas especiais que atenuam o feixe de raios $\mathrm{X}$, fabricadas com outros materiais absorvedores diferentes do chumbo e que não diminuem a sensibilidade das mãos ${ }^{(\mathbf{1 2})}$.
Recomenda-se que neste serviço os médicos intervencionistas sejam monitorados nas mãos, mediante o uso de dosímetros adicionais, tais como os dosímetros de pulso, principalmente durante os procedimentos realizados por via braquial e no modo contínuo de fluoroscopia.

\section{REFERÊNCIAS}

1. International Commission on Radiological Protection. Avoidance on radiation injuries from medical interventional procedures. ICRP Publication No. 85. Ann ICRP. 2000;30:7-67.

2. Padovani R, Rodella CA. Staff dosimetry in interventional cardiology. Radiat Prot Dosimetry. 2001;94:99-103.

3. Haskal ZJ, Worgul BV. Interventional radiology carries occupational risk for cataracts. RSNA News. 2004;14:5-6.

4. International Commission on Radiological Protection. Recommendations of the International Commission on Radiological Protection. ICRP Publication No. 60. Oxford: Pergamon Press; 1991.

5. Brasil. Ministério da Saúde. Diretrizes de proteção radiológica em radiodiagnóstico médico e odontológico. Portaria $n^{\circ}$ 453. Brasília: Diário Oficial da União $\mathrm{n}^{\circ} 100,1 / 6 / 1998$.

6. International Commission on Radiological Protection. Radiological protection and safety in medicine. ICRP Publication No. 73. Ann ICRP. 1996;26:1-47.

7. Comissão Nacional de Energia Nuclear. Diretrizes básicas de radioproteção. Resolução CNEN $n^{\circ}$ 27/2004, Norma CNEN-NN-3.01. Rio de Janeiro: CNEN; 2004.

8. Maurício CLP. Noções de dosimetria termoluminescente: aplicação em dosimetria individual [apostila]. Rio de Janeiro: CNEN; 2001.

9. Sandborg M, Fransson SG, Pettersson H. Evaluation of patient-absorbed doses during coronary angiography and intervention by femoral and radial artery access. Eur Radiol. 2004;14:653-8.

10. Tauhata L, Salati I, Di Prinzio R, et al. Fundamentos de radioproteção e dosimetria [apostila]. Rio de Janeiro: CNEN; 2003.

11. Luz ES, Canevaro LV, Ferreira NMPD, et al. A importância do controle de qualidade em serviços de hemodinâmica e cardiologia intervencionista. Radiol Bras. 2007;40:27-32.

12. Marx MV, Ellis JH. Radiation protection of the hand in interventional radiology: should it fit like a glove? Radiology. 1996;200:24-5. 